\title{
Evaluation of response to chemotherapy in locally advanced breast cancer with mri, importance of lesion size and adc value, and association with ki-67 index
}

\author{
Cengiz $A^{1}$, Altunkeser $A^{1 *}$, Eryilmaz MA $^{2}$, Tolu $I^{1}$ and Kucukosmanoglu $I^{3}$ \\ ${ }^{1}$ Department of Radiology, University of Health Science, Konya Trainning and Research Hospital, Konya, Turkey \\ ${ }^{2}$ Department of General Surgery, University of Health Science, Konya Trainning and Research Hospital, Konya, Turkey \\ ${ }^{3}$ Department of Pathology, University of Health Science, Konya Trainning and Research Hospital, Konya, Turkey
}

\begin{abstract}
Introduction: Contrast-enhanced and diffusion-weighted magnetic resonance imaging is one of the most sensitive imaging techniques used to diagnose and manage breast cancer. Neoadjuvant chemotherapy is applied to the patients before surgery in locally advanced breast cancer. To investigate the role of contrast-enhanced and diffusion-weighted magnetic resonance imaging in evaluating neoadjuvant chemotherapy response in locally advanced breast cancer, we compared the pre-and posttreatment lesion size and $\mathrm{ADC}$ values and evaluated their relationship with the Ki-67 index.

Material and Method: In the scope of the study, 39 female patients with locally advanced breast cancer who underwent neoadjuvant chemotherapy and surgical operation between 2010 and 2017 were retrospectively reviewed. Lesion size was determined from contrasted magnetic resonance imaging before and after neoadjuvant chemotherapy, ADC value was measured from diffusion weighted images and these were compared. The size measured after neoadjuvant chemotherapy was compared with the surgically removed tumor size. The relationship between tumor size and ADC value before the treatment and Ki- 67 index was investigated.

Result: Lesion size which was measured with magnetic resonance imaging after neoadjuvant chemotherapy was correlated with surgically removed tumor size. Significant reduction in tumor volume and increase in ADC value were observed with treatment $(\mathrm{p}<\mathbf{0 . 0 0 1})$. Pretreatment ADC values of tumors with high Ki-67 index were low $(\mathbf{p}=\mathbf{0 . 0 3 1})$, while ADC values of surgically removed large lesions were high $(\mathrm{p}<\mathbf{0 . 0 0 1})$.
\end{abstract}

Conclusion: Tumor size obtained with magnetic resonance imaging and ADC value are important parameters to follow response to neoadjuvant chemotherapy in locally advanced breast cancer. It may also guide treatment management if used combined with $\mathrm{Ki}-67$ index.

\section{Introduction}

Contrast and diffusion-weighted magnetic resonance imaging (C-DW-MRI) is an important imaging method that has a high sensitivity in detecting invasive breast cancer and gives information about lesion morphology, contrast involvement and cell density [1,2]. Local advanced breast cancer (LABC) is used to define clinical stage III breast cancer and neoadjuvant chemotherapy (NAC) is used for its treatment to increase chance of breast protecting surgery [3]. Preoperative NAC is also important as an indicator that predicts response to treatment at postoperative period. For this reason, it is important to calculate the tumor size correctly in a patient treated with NAC, since it will be a treatment response indicator. Today, breast MRI has an important role to measure tumor size with highest correctness [4]. According to a limited number of recent studies, it has been suggested that breast MRI detects residual tumor tissue size after NAC with higher accuracy than conventional imaging and clinical evaluation $[5,6]$.

$\mathrm{Ki}-67$ is an antigen found in cell nucleus which acts as a pathological prognostic parameter that gives information about cellular proliferation in tumoral tissue. In tumors with fast cell proliferation, the tumor is aggressive and the Ki-67 index is high. Ki-67 elavation also allows us to predict that rapidly growing malignant cells will benefit more from NAC [7-9].
We wanted to investigate the role of C-DW-MRI in assessing NAC response in patients diagnosed with LABC, as early and accurate detection of chemotherapy response in patients receiving NAC would provide important information about the efficacy of the treatment. For this purpose, we measured lesion size and ADC (Apparent diffusion coefficient) values of patients diagnosed with LABC using C-DW-MRI before and after NAC, and we compared measured tumor size and $\mathrm{Ki}$ 67 index using the specimens obtained after surgery.

\section{Material and method}

We included 39 female patients who were diagnosed as locally advanced breast cancer between 2010 and 2017, received NAC, and operated after the treatment to our study. For our retrospective study, approval was obtained from Health Sciences University Konya Research and Education Hospital Local Ethics Board with a decision number of

${ }^{*}$ Correspondence to: Altunkeser A, Konya Education and Research Hospital, Meram Yeni Yol Konya, Turkey, Mobile: +905335507400; Tel: +903323236709; Fax: +903323236723; E-mail: aaltunkeser@hotmail.com

Key words: Contrast-enhanced and diffusion-weighted magnetic resonance imaging, locally advanced breast cancer, neoadjuvant chemotherapy, Ki-67

Received: May 11, 2018; Accepted: May 23, 2018; Published: May 28, 2018 
2214/5 and all the study was conducted in accordance with Helsinki Declaration. According to WHO criteria;

* Patients with a T3 tumor greater than $5 \mathrm{~cm}$ and have mobile lymph nodes palpated at ipsilateral axilla.

* Stage T4a and T4b patients with invasion to chest wall regardless of tumor size,

* Patients at stage N2a with ipsilateral axillary lymph nodes fixed to one another,

* Patients at stage N2b and internal mammary lymph node involvement without axillary metastasis,

* Patients at stage N3b and ipsilateral involvement in axillary and internal mammary lymph nodes,

* Patients at stage N3a and N3c with ipsilateral infra or supraclavicular lymph node involvement, patients with most stage II breast cancers that are operable but tumor sizes are big relative to breast and patients with stage IIb breast cancers were accepted as the LABC patient group. Patients who had previous history of breast cancer operation, had another malignancy, or were unable to receive NAC treatment for any reason were excluded from the study. In all cases, C-DW-MRI was performed before and after NAC in our clinic.

\section{MR Protocol}

Breast C-DW-MRIs were performed in a supine position using a 16-channel breast coil with a 1.5 Tesla device (MAGNETOM Avanto, Siemens, Erlangen, Germany). Image protocol was started with bilateral, consecutive, Coronal flash2d-grappa (TR/TE: 160/4.5, matrix: 352x384, section thickness: $3 \mathrm{~mm}$, FOV: $180-500 \mathrm{~mm}$ ) and axial turbospinecho (TR/TE: 417/11, matrix: 352x384, section thickness: 3 mm, FOV: 280 $300 \mathrm{~mm}$ ) T1A sequences. Then T2A TIRM (TR / TE: 2770/66 ms, matrix: 352x384, inversion time: $150 \mathrm{~ms}$, flip angle: 150 degrees, spatial resolution: $0.7 \times 0.7 \times 2 \mathrm{~mm}$, acquisition time: 3 minutes 26 seconds) and DWI using a planar sequence (TR / TE: 6200/88, spatial resolution: 2.7x2.7x4 mm, cross-sectional thickness: $3 \mathrm{~mm}$, FOV: $258-300 \mathrm{~mm}, \mathrm{~B}$ values 0 and $800 \mathrm{~s} / \mathrm{mm}^{2}$, spectral fat saturation and acquisition time: 2 minutes 47 seconds) were obtained. Subsequently, dynamic study first was performed without contrast and then by giving $0.1 \mathrm{mmol}$ of gadolinium chelates per $\mathrm{kg}$ by infusion pump and then obtaining 5 times T1-gradient gradient echo3D FLASH (fast low angle shot) sequence (TR / TE: $4.79 / 1.70 \mathrm{msn}$, spatial resolution: $0.8 \times 0.8 \times 1.3$ $\mathrm{mm}$, cross-sectional thickness: $1.6 \mathrm{~mm}$, FOV: $318-500 \mathrm{~mm}$ ). All early and late sections with contrast were subtracted from sections without contrast using device's standard subtraction function and subtraction images were obtained.

\section{Image analysis}

C-DWI-MRI's of the patients were re-evaluated before and after NAC. In the evaluation lesion size was measured by selecting the section in which the lesion can be defined the best in dynamic evaluation and ADC values were obtained by ROI from the ADC map.

\section{Histopathological assessment}

Postoperative pathology reports of the patients were retrospectively reviewed from the hospital records. Histopathological evaluations were performed by a pathologist experienced in breast histopathology from the preparations obtained after surgery. Tumor size, axillary lymph node status, shape, border properties, multicentricity, and lymphovascular invasion status were defined as the classic prognostic factors. Ki-67 index was defined as the main pathological prognostic parameter. During evaluation, according to the percentage of cells that are stained positively in Ki-67, 20\% and above were defined as strong positive. The whole postoperative surgical material was evaluated.

\section{Statistical analysis}

Statistical Package for Social Sciences (SPSS) 15 software was used for statistical analysis. In addition to the descriptive statistical methods (Mean, Standard Deviation, Median, Frequency, Ratio) the Chi-Square test was used to compare two groups with normal distribution and to identify the group that caused the difference in the comparison of the quantitative data. $\mathrm{P}<0.05$ was considered statistically significant.

\section{Result}

The mean age of the $39 \mathrm{LABC}$ cases included in the study was 48 (28-75 years). The mass size measured at C-MRI after NAC was in accordance with the size of the surgically removed mass. The median tumor mass dimensions after NAC and surgical excision were 0.8 and 1 $\mathrm{cm}$, respectively (Tables $1 \mathrm{a}, 1 \mathrm{~b}$ ), (Figure 1 ).

Tumor sizes and ADC values of the patients measured with C-DWMRI before and after NAC are presented in Table 1a. The median size of the mass measured in C-DWMRI before NAC of the examined patients was $3.5 \mathrm{~cm}$, whereas it was $0.8 \mathrm{~cm}$ after NAC $(\mathrm{p}<0.001)$. Median ADC value before NAC was 0.9 (min: 0.6-max: 1.3 ), and after NAC was 1.2 (min: 1-Max: 1.7) $(\mathrm{p}<0.001)$. Decrease in mass size and increase in ADC value before and after NAC were statistically significant.

Size of the tumor obtained after surgery, histopathological type and $\mathrm{Ki}-67$ index value are presented in Table $1 \mathrm{~b}$ (Figure 2a-b). The median $\mathrm{Ki}-67$ index of the patients was calculated as 20 (min: 6-max: 80). The median tumor size obtained after surgery was 1 (min: 0 -max: 4$) \mathrm{cm}$. Invasive ductal carcinoma was present in $92.3 \%(n=36)$ of the lesions in the pathological subtyping, whereas invasive lobular carcinoma was present in $7.7 \%(\mathrm{n}=3)$.

The relationship between size of the tumor after surgery and Kiindex with tumor size before NAC and ADC are shown in Table 1c (Figure 3a-b). A positive correlation was detected between tumor size after surgery and ADC value before NAC $(\mathrm{r}=0.57 ; \mathrm{p}<0.001)$. No

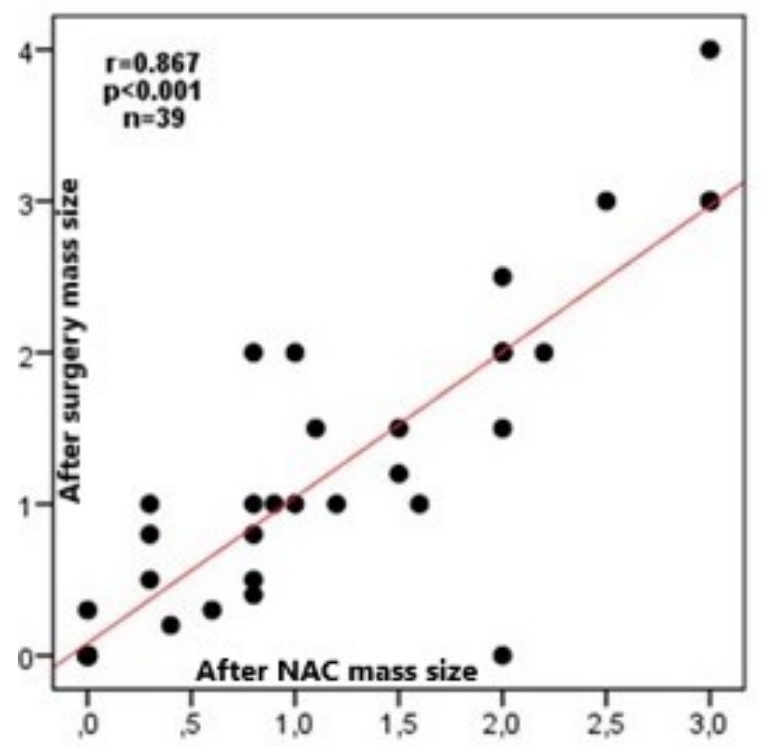

Figure 1. Relationship between tumor size obtained after surgery and lesion size measured by MRI after NAC 


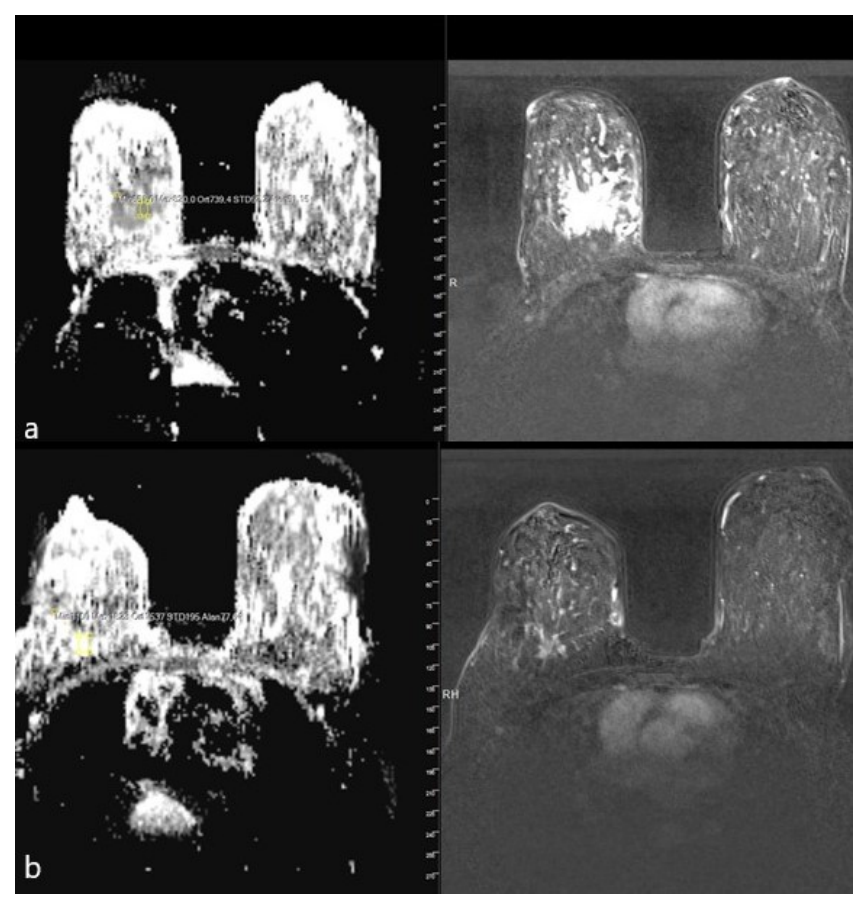

Figure 2a-b. ADC mapping and $\mathrm{T} 1$ weighted subtraction imaging of a 45-year old case with $\mathrm{LABC}$ detected to have invasive ductal carcinoma at right breast. a) A $57 \mathrm{x} 45 \times 38 \mathrm{~mm}$ mass lesion with a spiculated contour on MRI and a mean ADC value of 0.75 before NAC b) After NAC, mass dimension was reduced to $25 \times 20 \times 20 \mathrm{~mm}$ and the mean ADC value increased to 1.5 . Surgically removed tumor size and size measured on MRI after NAC were similar and $25 \times 18 \times 20 \mathrm{~mm}$

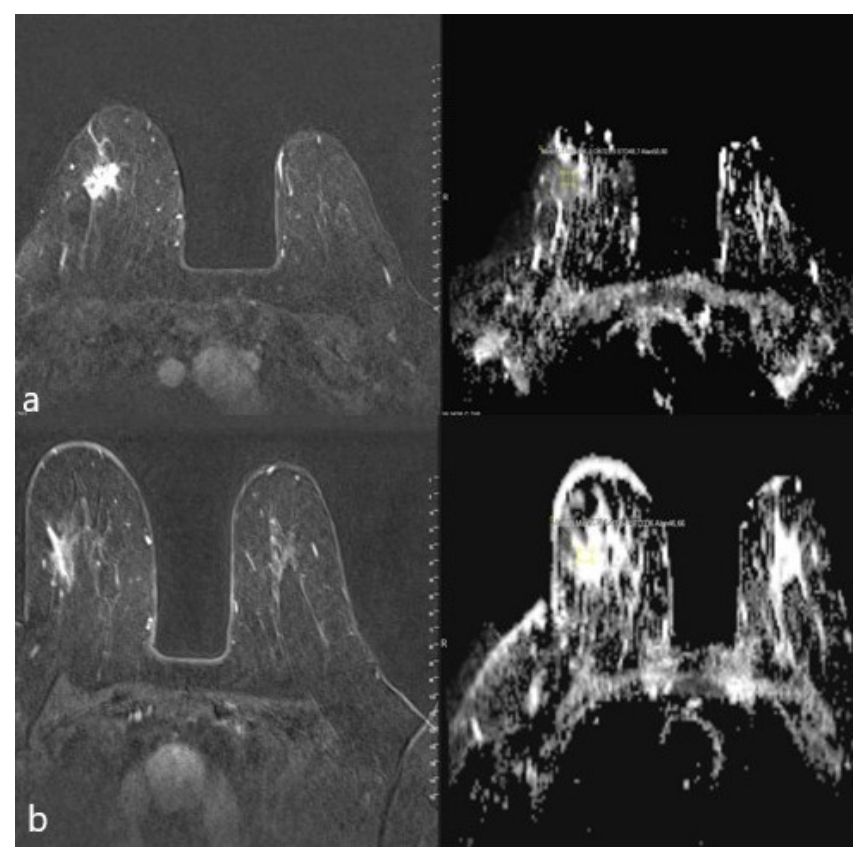

Figure 3a-b. ADC mapping and T1 weighted subtraction imaging of a 66-year-old case detected breast invasive ductal carcinoma with a mass of 22x16x19 mm with speculated contour; a) Mean ADC value before NAC was 0.72 and b) Mean ADC after NAC was 1.7

statistically significant relationship could be detected between tumor size before NAC and after surgery ( $p>0.05$ ). There was a moderate negative correlation between Ki-67 index obtained from tumor tissue after surgery and ADC value before NAC $(\mathrm{r}=-0.34 ; \mathrm{p}<0.05)$. On the otherhand no statistically significant association could be detected between $\mathrm{Ki}-67$ index and tumor size measured before NAC ( $\mathrm{p}>0.05)$ (Table 1c).

\section{Discussion}

The high sensitivity of contrast-enhanced MRI to post-NAC lesion size is a superiority of MRI over other conventional methods [10,11]. This superiority suggests that it is the effective imaging modality that can be used to evaluate effectiveness of antitumor chemotherapeutic agents that decrease tumor size and to predict prognosis. The vast majority of prognostic factors in treatment strategy are pathological prognostic factors and there are not sufficient number of established radiological prognostic factors. In our study, we evaluated decrease in tumor size, which is a radiological prognostic factor with C-MRI, and measured ADC from DWI, and investigated their association with Ki67 index which is an established pathological prognostic factor. The most important expectation in the treatment of LABC is to decrease local recurrence rate and status of surgical border positivity which will require re-excision. This expectation can be achieved by radiologically determining the decrease in tumor size after NAC with accuracy. In our study, we detected that C-MRI can give tumor volume after NAC with high accuracy and there was not a significant difference between lesion size calculated by C-MRI and surgically excised lesion size. For this reason, we suggest that C-MRI is a radiological modality that can accurately show margins of lesion that should be excised surgically. Research has indicated that C-MRI has accuracy reaching $90 \%$ to show the dimensions of recurrent tumor, because of having high spatial resolution, demonstration of contrasting patterns of the lesion and enabling three-dimensional measurement $[7,12]$. Although many studies have detected that it can determine residual tumor size with a higher accuracy than physical examination and conventional methods [13-15], Rieber et al stated that C-MRI has high false negativity rate in cases receiving chemotherapy and it may be insufficient and misleading to calculate residual tumor volume $[16,17]$. Although some studies have false positive and false negative results, current literature suggests that adding ADC mapping to C-MRI increases specificity [18].

Table 1a. Mass size and ADC value measured before and after NAC with C-DW-MRI

\begin{tabular}{|c|c|c|c|}
\hline \multirow{2}{*}{$(\mathbf{n}=\mathbf{3 9})$} & Before NAC & After NAC & \multirow{2}{*}{} \\
\cline { 2 - 3 } & $\begin{array}{c}\text { mean } \pm \text { SD } / \text { median } \\
(\min -\max )\end{array}$ & $\begin{array}{c}\text { mean } \pm \mathrm{SD} / \text { median } \\
(\min -\max )\end{array}$ & \multirow{2}{*}{} \\
\hline $\begin{array}{c}\text { Mass Dimension } \\
(\mathbf{c m})\end{array}$ & $\begin{array}{c}3.60 \pm 1.28 / 3.5 \\
(1.5-7.0)\end{array}$ & $\begin{array}{c}1.06 \pm 0.95 / 0.8 \\
(0-3.0)\end{array}$ & $<\mathbf{0 . 0 0 1}$ \\
\hline ADC $\left(\mathbf{1 0}^{-\mathbf{3}} \mathbf{~ m m}^{\mathbf{2}} / \mathbf{s n}\right)$ & $\begin{array}{c}0.9 \pm 0.17 / 0.9(0.6- \\
1.3)\end{array}$ & $\begin{array}{c}1.253 .8 \pm 0.2101 / \\
1.2(1-1.7)\end{array}$ & $<\mathbf{0 . 0 0 1}$ \\
\hline
\end{tabular}

NAC- Neoadjuvant chemotherapy; ADC- Apparent diffusion coefficient; n- Number of patients; m- Mean; SD- Standard deviation; *Wilcoxon Signed Rank Test

Table 1b. Ki-67 index value, size and subtypes of tumor tissue after surgery

\begin{tabular}{|l|c|}
\hline Pathology Results $(n=39)$ & mean \pm SD $/$ median (min-max) \\
\hline Ki-67 index & $27.79 \pm 19.19 / 20(6-80)$ \\
\hline Mass Dimension (cm) & $1.10 \pm 1.05 / 1(0-4)$ \\
\hline Subtypes & $\mathbf{n}(\mathbf{\%})$ \\
\hline \multicolumn{1}{|c|}{ IDC } & $36(92.3)$ \\
\hline ILC & $3(7.7)$ \\
\hline
\end{tabular}

Table 1c. Relationship between post-surgical tumor size and Ki-67 index with mass size measured with C-DW-MRI and ADC values before NAC

\begin{tabular}{|l|l|c|c|c|}
\hline$(n=39)$ & & Pathology-Size (cm) & Ki-67 \\
\hline Before NAC & & & \\
\hline \multirow{2}{*}{ MRI-Size (cm) } & r & p & 0.246 & -0.188 \\
\cline { 2 - 5 } & & & $0.867 / 0.004$ & $-0.345 /-0.328$ \\
\hline \multirow{2}{*}{ ADC } & r & p & $<\mathbf{0 . 0 0 1} / 0.982$ & $\mathbf{0 . 0 3 1 / 0 . 0 4 2}$ \\
\hline
\end{tabular}

NAC-Neoadjuvant chemotherapy; n- Number of patients; r- Spearman Correlation Coefficient; ADC- Apparent diffusion coefficient 
In diffusion-weighted imaging $\mathrm{ADC}$ value decreases in tumoral tissues with dense cellular structures and helps to diagnose breast cancer correctly. Studies conducted in recent years indicate that ADC value is useful for showing the treatment response in malignant lesion. Study by Nilsen et al in LABC cases emphasized that ADC values increase significantly after 4 cycles of NAC [19]. Sharma et al [14] compared morphological parameters and ADC values in patients and control group. They stated that ADC values increased significantly in tumoral tissue after NAC and this increase was more important than morphological parameters. Yankeelov et al suggested that [13] ADC mapping should be done before and after NAC in LABC cases and ADC is a sensitive indicator that shows early tumor response. Our study also supported the current literature as the increase in ADC values after NAC was significant. Decrease in tumor size and increase in ADC that we detected were directly associated with NAC. Probably chemotherapeutic agents that trigger apoptosis in tumoral tissue cause destruction of cellular wall which enlarges extracellular space and augments water diffusion. Another important finding of our study was the significant positive correlation between the tumor size obtained after surgery and ADC values before NAC. This finding may mean that tumors with high ADC values before NAC may have a lower treatment response.

In multivariate analyzes of survival and prognosis in breast cancer, Ki-67 index before and after NAK was found to be a significant independent predictor. In patients who lack a pathological complete response, $\mathrm{Ki}-67$ index after NAC is said to be a very powerful prognostic marker [20]. Increased Ki-67 index which is an indicator of fast cell turnover has been shown in basal-like breast cancers which have aggressive features and bad prognosis [21]. In our study, we detected a negative correlation between ADC values before NAC and Ki-67 index. Increased cell density of tumors that have high $\mathrm{Ki}-67$ may be the reason for decreased ADC in tumoral tissue before NAC. Low ADC value before NAC may indicate that the tumor will be aggressive and also a good treatment response will be obtained. We suggest that ADC value may be used to determine prognosis, and to predict and follow NAC response.

In conclusion, contrast MRI measures tumor size after NAC similar to pathological tumor size. A decrease in tumor size and increase in ADC values are seen in LAMC cases taking neoadjuvant therapy. In tumors with a high ADC value before NAC, the size reduction is small, and the Ki-67 index is high in tumors with low ADC value. ADC value in LABC cases is important to determine prognosis and to predict and follow NAC response; it is a candidate to be a prognostic marker to guide chemotherapeutic drugs which have toxic effects. However, there is also a need for prospective large-scale studies on this subject.

\section{Financial support}

None

\section{Declaration of interest}

The authors report no conflict of interest.

\section{Acknowledgement}

None

\section{References}

1. Orel SG (2001) MR imaging of the breast. Magn Reson Imaging Clin N Am 9: 273-288. [Crossref]

2. Woodhams R, Ramadan S, Stanwell P, Sakamoto S, Hata H, et al. (2011) Diffusionweighted imaging of the breast: principles and clinical applications. Radiographics 31 : 1059-1084. [Crossref]
3. Güler N, Karabulut B, Koçdor MA, Kaya H, Esen G, et al. (2011) Local advanced breast cancer 2010 istanbul breast cancer consensus meeting. $J$ Breast Health 7.

4. Daniel BL, Ikeda DM (2004) Magnetic resonance imaging of breast cancer and MRIguided biopsy. In: Ikeda DM Ed. The requisites: Breast Imaging. Philadelphia: Elsevier Mosby 189-224.

5. Nunes LW, Schnall. Orel SG (1999) Correlation of lesion appearance and histologic findings for the nodes of a breast MR imaging interpretation model. Radiographics 19: 79-92.

6. Rausch DR, Hendrick RE (2006) How to optimize clinical breast MR imaging practices and techniques on Your 1.5-T system. Radiographics 26: 1469-1484. [Crossref]

7. Wright H, Listinsky J, Rim A, Chellman-Jeffers M, Patrick R, et al. (2005) Magnetic resonance imaging as a diagnostic tool for breast cancer in premenopausal women. The American Journal of Surgery 190: 572-575

8. Yabuuchi H, Matsuo Y, Okafuji T, Kamitani T, Soeda H, et al. (2008) Enhanced mass on contrast- enhanced breast MR imaging: lesion characterization using combination of dynamic contrast-enhanced and diffusion-weighted MR images. J Magn Reson Imaging 28: 1157-1165. [Crossref]

9. Hatakenaka M, Soeda H, Yabuuchi H, Matsuo Y, Kamitani T, et al. (2008) Apparen diffusion coefficients of breast tumors: clinical application. Magn Reson Med Sci 7: 23-29. [Crossref]

10. Drew PJ, Chatterjee S, Turnbull LW, Read J, Carleton PJ, et al. (1999) Dynamic contras enhanced magnetic resonance imaging of the breast is superior to triple assessment for the pre-operative detection of multifocal breast cancer. Ann Surg Oncol 6: 599-603. [Crossref]

11. Fornage BD, Toubas O, Morel M (1987) Clinical, mammographic, and sonographic determination of preoperative breast cancer size. Cancer 60: 765-771. [Crossref]

12. Manton DJ, Chaturvedi A, Hubbard A, Lind MJ, Lowry M, et al. (2006) Neoadjuvant chemotherapy in breast cancer: early response prediction with quantitative MR imaging and spectroscopy. Br J Cancer 94: 427-435. [Crossref]

13. Yankeelov TE, Lepage M, Chakravarthy A, Broome EE, Niermann KJ, et al. (2007) Integration of quantitative DCE-MRI and ADC mapping to monitor treatment response in human breast cancer: initial results. Magn Reson Imaging 25: 1-13. [Crossref]

14. Sharma U, Danishad KK, Seenu V, Jagannathan NR (2009) Longitudinal study of the assessment by MRI and diffusion-weighted imaging of tumor response in patients with locally advanced breast cancer undergoing neoadjuvant chemotherapy. NMR in Biomedicine 22: 104-113. [Crossref]

15. Rosen EL, Blackwell KL, Baker JA, Soo MS, Bentley RC, et al. (2003) Accuracy of MRI in the detection of residual breast cancer after neoadjuvant chemotherapy. AJR Am J Roentgenol 181: 1275-1282. [Crossref]

16. Rieber A, Zeitler H, Rosenthal H, Görich J, Kreienberg R, et al. (1997) MRI of breast cancer: influence of chemotherapy on sensitivity. Br J Radiol 70: 452-458. [Crossref]

17. Rieber A, Brambs HJ, Gabelmann A, Heilmann V, Kreienberg R, et al. (2002) Breast MRI for monitoring response of primary breast cancer to neo-adjuvant chemotherapy. Eur Radiol 12: 1711-1719. [Crossref]

18. Song SE, Park EK, Cho KR, Seo BK, Woo OH, et al. (2017) Additional value of diffusion-weighted imaging to evaluate multifocal and multicentric breast cancer detected using pre-operative breast MRI. Eur Radiol 27: 4819-4827. [Crossref]

19. Nilsen L, Fangberget A, Geier O, Olsen DR, Seierstad T (2010) Diffusion-weighted magnetic resonance imaging for pretreatment prediction and monitoring of treatment response of patients with locally advanced breast cancer undergoing neoadjuvant chemotherapy. Acta Oncol 49: 354-60. [Crossref]

20. Jones RL, Salter J, A'Hern R, Nerurkar A, Parton M, et al. (2009) The prognostic significance of Ki67 before and after neoadjuvant chemotherapy in breast cancer. Breast Cancer Res Treat 116: 53-68. [Crossref]

21. Osborne CR KL, Xie XJ, Ashfaq R, Bian A, Tripathy D (2006) Neoadjuvant chemotherapy for basal-like breast cancer cohort: clinical and pathological outcomes. Breast Cancer Res Treat 100: S53.

Copyright: (C2018 Cengiz A. This is an open-access article distributed under the terms of the Creative Commons Attribution License, which permits unrestricted use, distribution, and reproduction in any medium, provided the original author and source are credited. 